\title{
A Novel Basalt Flake Epoxy Resin Coating Modified by Carbon Nanotubes
}

\author{
Lida Luo ${ }^{1,2}$, Qihui Wang ${ }^{3}$, Qian Ma ${ }^{1,2}$, Qingwei Wang ${ }^{1,2, *}$, Jin Liu ${ }^{1,2}$, Linfeng Ding ${ }^{1,2, *(\mathbb{C}}$ and \\ Weizhong Jiang ${ }^{1,2, *}$ \\ 1 State Key Laboratory for Modification of Chemical Fibers and Polymer Materials, Donghua University, \\ Shanghai 201620, China; 1ld@dhu.edu.cn (L.L.); 2170444@mail.dhu.edu.cn (Q.M.); lj@dhu.edu.cn (J.L.) \\ 2 Engineering Research Center of Advanced Glass Manufacturing Technology, Ministry of Education, \\ Donghua University, Shanghai 201620, China \\ 3 Shanghai Chengjian NichiReki Special Asphalt Co., Ltd., Shanghai 200436, China; \\ wangqihui2020@aliyun.com \\ * Correspondence: wqwq888@dhu.edu.cn (Q.W.); linfeng.ding@dhu.edu.cn (L.D.); jwzh@dhu.edu.cn (W.J.)
}

Received: 19 September 2019; Accepted: 26 October 2019; Published: 31 October 2019

\begin{abstract}
As a new type of anticorrosive material, basalt flakes (BFs) have been widely used in the marine industry due to their good acid and alkali corrosion resistance and dispersion stability. In this work, the effect of carbon nanotubes (CNTs) addition on properties improvement of basalt flake epoxy resin (CNT-BF/EP) coating was studied. Firstly, $0-0.7 \mathrm{wt} . \%$ acidified CNTs was used to modify the surface of the BFs and the CNT-BF/EP coating was successfully prepared. Experimental results showed that the performance of the interfacial compatibility, the tensile strength, and acid and alkali resistance of the CNT-BF/EP coating were significantly improved with the addition of the CNTs. Particularly, the CNT-BF/EP coating achieved the best comprehensive properties (tensile strength increased to $30.3 \mathrm{MPa}$, surface weight loss rate of only $1.0 \mathrm{wt} . \%$ in the acid environment for $480 \mathrm{~h}$, and water absorption of only $1.1 \mathrm{wt} . \%$ after $480 \mathrm{~h}$ ) when the CNTs addition reached $0.5 \mathrm{wt} . \%$. This work suggests a feasible way to enhance the mechanical properties and chemical durability of the basalt flakes coating.
\end{abstract}

Keywords: basalt flake; carbon nanotubes; coating; tensile strength; chemical durability

\section{Introduction}

Nowadays, the corrosion of ships, wharves, and various marine engineering equipment has attracted more and more attention [1]. The application of coatings becomes more extensive in the marine industry due to its advantages such as simple operation, low cost, and good anti-corrosion effect.

Flake anticorrosive coatings are widely used in the marine industry because of their high efficiency, simple construction, strong adhesion, and good corrosion resistance [2]. Glass flake (GF) is one of the first kinds of materials studied and used in the area of flake anticorrosive coatings [3,4]. In addition, graphite flakes and mica flakes anticorrosive coatings are also widely applied [5,6]. There are three main functions of flakes in the coatings: (1) flakes can enhance the chemical durability of the coatings; (2) flakes acting as fillers can enhance the mechanical strength of the coatings; (3) flakes usually have a large diameter-thickness ratio, which could form the "labyrinth effect" due to the staggered parallel arrangement in the resin. Broughton et al. [7] demonstrated that GFs modified with aminosilane exhibited higher stiffness and strength than GFs modified with titanate.

Basalt flake (BF), as a new type of inorganic material with advantages including low cost and excellent shielding performance, has gradually gained people's research interests. In addition, BF has attracted wider attention because of its better acid and alkali resistance and more uniform particle size 
distribution than GF [8]. Yan et al. [9] studied the alkali and acid resistance of basalt flake-epoxy coatings and concluded that good chemical durability improves the anticorrosive property of epoxy coatings. Luo et al. [8] used the nano- $\mathrm{SiO}_{2}$ microspheres to modify the BF-epoxy coating and demonstrated the feasibility of using nano-SiO $\mathrm{S}_{2}$ microspheres to modify $\mathrm{BF}$ epoxy resin coating and enhance the chemical durability and mechanical properties provided by the coating.

Inorganic fillers should be pretreated to solve the problems of large polarity difference and poor interfacial compatibility [10] during coatings preparation. The common surface treatment methods of inorganic fillers are as follows: (1) adding silane coupling agents; (2) nano- $\mathrm{SiO}_{2}$ modifying [8]; (3) surface grafting; (4) acid and alkali etching treatments [11-13].

Carbon nanotubes (CNTs) have excellent mechanical properties [14,15], and thus, are ideal for interfacial modification and reinforcing materials. Studies have shown that the modification of inorganic fibers such as glass fibers, basalt fibers, and carbon fibers with CNTs can improve the interfacial properties of fibers and resin matrix, and therefore, significantly improve the mechanical properties of materials.

The surface modification of BFs and its application in coatings are still in the initial stage. The purpose of this paper is to improve the compatibility between BFs and epoxy resin and further enhance the properties of BFs epoxy resin coating. In this study, modified BFs epoxy resin coating was prepared with different content of acidified carbon nanotubes (CNTs). The chemical durability and mechanical properties of CNTs-BFs epoxy resin coating were studied.

\section{Experimental Procedures}

\subsection{Materials}

The basalt flakes (BFs) (Jiangsu Tianlong Continous Baslt Fiber Co., Ltd., Jiangsu, China) used in this work had an average thickness of $5 \pm 2 \mu \mathrm{m}$, length of 10-50 $\mu \mathrm{m}$, and a density of approximately $3.0 \mathrm{~g} / \mathrm{cm}^{3}$. The multi-wall carbon nanotubes (MWCNTs) (YusLab Technology Co., Ltd., Chongqing, China) had an average diameter of $20-30 \mathrm{~nm}$, length of $10-20 \mu \mathrm{m}$, and purity of $98 \%$. All the other chemicals used in this work were AR grade and supplied by Sinopharm Chemical Reagent Co., Ltd, Shanghai, China.

\subsection{Sample Preparation}

Firstly, we prepared the acidified CNTs. One gram of CNTs were added in a mixed acid solution $\left(V_{\mathrm{H}_{2} \mathrm{SO}_{4}}: V_{\mathrm{HNO}_{3}}=75 \mathrm{~mL}: 25 \mathrm{~mL}\right)$ and stirred for $3 \mathrm{~h}$ at $60^{\circ} \mathrm{C}$. Then, the $\mathrm{CNTs}$ were washed with deionized water and acetone solution. Afterward, the CNTs were filtered and dried to obtain acidified CNTs.

Secondly, we pre-activated BFs. The silane coupling agent KH560 (5 g) was added to an alcohol solution $\left(V_{\mathrm{H}_{2} \mathrm{O}}: V_{\mathrm{C}_{2} \mathrm{H}_{5} \mathrm{OH}}=10 \mathrm{~mL}: 40 \mathrm{~mL}\right)$, and the $\mathrm{pH}$ of the solution was adjusted to 3-4 with acetic acid to get solution $\mathrm{A}$. Then, $10 \mathrm{~g}$ BFs were dispersed in $250 \mathrm{~mL}$ xylene solution to obtain a mixture $\mathrm{B}$. Afterward, solution A was added to solution B and stirred continuously for $3 \mathrm{~h}$ at $80^{\circ} \mathrm{C}$ and $1 \mathrm{~h}$ at $110^{\circ} \mathrm{C}$. Finally, the pre-activated BFs was obtained after washing and drying.

Thirdly, we modified the pretreated BFs by acidified CNTs. The pretreated KH560-BFs (25 wt.\% of coating quality) were mixed in acetone solution and stirred for $20 \mathrm{~min}$ to obtain solution $\mathrm{C}$. Then, 0-0.7 wt.\% acidified CNTs were added to solution C and stirred for $60 \mathrm{~min}$ to obtain solution D. The CNTs-modified BFs were obtained by centrifuging, washing, and drying of the solution $\mathrm{D}$, which were named as 0.1 CNT-BF, 0.3 CNT-BF, 0.5 CNT-BF, and 0.7 CNT-BF, respectively.

Lastly, we prepared the CNT-BF epoxy coating. In the BF epoxy coating, the solvent of xylene and n-butanol (mass ratio of 7:3) accounts for $20 \mathrm{wt} . \%$ of the total mass of the coating, and the epoxy resin E-51 and 593 curing agents (mass ratio of 4:1) account for $80 \mathrm{wt} . \%$ of the total mass. In addition, an antifoaming agent with a total coating mass of $0.5 \%$ was added. The BF epoxy resin solution was stirred for $40 \mathrm{~min}$ and added $25 \mathrm{wt} . \%$ of CNT-BF with continuously magnetic stirring for $60 \mathrm{~min}$. 
Finally, the CNT-BF epoxy resin (CNT-BF/EP) coating was obtained by pouring the mixture into a custom polytetrafluoroethylene mold and solidifying at room temperature for $24 \mathrm{~h}$.

\subsection{Characterization Techniques}

The chemical structure of the CNTs was measured by applying Fourier transform infrared (FT-IR, Nexus-670, Thermo Fisher Scientific, Waltham, MA, USA) spectra, where the CNTs samples were mounted in the $\mathrm{KBr}$ pelletized disks and the surface of samples were measured in the $400-4000 \mathrm{~cm}^{-1}$ region with a resolution of $2 \mathrm{~cm}^{-1}$. The morphologies of the CNTs, BFs, and CNT-BF/EP samples were studied by scanning electron microscopy (SEM; SU8010, Hitachi, Tokyo, Japan). For the sample preparation, $0.05 \mathrm{~g}$ CNTs samples and $0.5 \mathrm{~g}$ BFs samples were added into $500 \mathrm{~mL}$ anhydrous ethanol solvent separately, dispersed by ultrasound for $20 \mathrm{~min}$, followed by dropping (2-3 drops) on the tin foil and dried for the measurement. The composite spline of CNT-BF/EP was frozen in liquid nitrogen and fractured, which was used to observe the section morphology. The CNT-BF/EP coating samples with dimensions of $75 \mathrm{~mm} \times 12.5 \mathrm{~mm} \times 2.0 \mathrm{~mm}$ were prepared for tensile strength measurements carried out using a Universal Testing Machine (WDW3020, Changchun Chuangyuan Testfacility Co., Ltd, Changchun, China) at a testing speed of $0.5 \mathrm{~mm} / \mathrm{min}$. The tensile strength $\sigma$ can be calculated by $\sigma$ $=F / A$, where $F$ is the maximum force, and $A$ is the original area of the cross-section.

\subsection{Chemical Durability and Water Absorption Tests}

The chemical durability of the modified CNT-BF/EP coating (15 $\mathrm{mm} \times 15 \mathrm{~mm} \times 5 \mathrm{~mm})$ was assessed by leaching tests [16] conducted at room temperature for $480 \mathrm{~h}$ in $3 \mathrm{~mol} / \mathrm{L} \mathrm{HCl}$ solution and $3 \mathrm{~mol} / \mathrm{L} \mathrm{NaOH}$ solution and shown by the surface weight loss rate. The surface weight loss rate $R_{1}$ can be calculated by $R_{1}=\left(m_{0}-m_{1}\right) / m_{0}$, where $m_{0}$ is the initial mass of the modified CNT-BF/EP coating, and $m_{1}$ is the mass after the leaching tests after washing by deionized water and drying. Each sample was measured five times by the Mettler Toledo AL204 balance (Mettler Toledo, Shanghai, China) at room temperature, and the results reported are the average values.

The water absorption of the CNT-BF/EP coating (50 mm diameter and $32 \mathrm{~mm}$ thickness) was assessed. First, the samples were immersed in deionized water at room temperature for 24, 240, and $480 \mathrm{~h}$. The samples were then taken out of the water, dried completely with cloth, and weighed using the Mettler Toledo AL204 balance (Mettler Toledo) as $m_{3}$. The water absorption rate $R_{2}$ can be calculated by $R_{2}=\left(m_{3}-m_{2}\right) / m_{2}[3,4]$, where $m_{2}$ is the initial weight of the modified CNT-BF/EP coating.

\section{Results}

\subsection{The FT-IR Analysis of CNTs}

The acid treatment can open five or seven-membered ring defects at the ports and introduce functional groups such as carboxyl $(-\mathrm{COOH})$ and hydroxyl $(-\mathrm{OH})$ into the surface of CNTs ports and sidewalls, which is conducive to its subsequent reaction [17]. It can be seen from Figure 1 that the peak strength of acidified CNTs was enhanced near $3490 \mathrm{~cm}^{-1}$ compared with the initial CNTs, which was induced by the stretching vibration of -OH caused by the presence of carboxyl group or water adsorbed on the surface [18]. The characteristic absorption peaks appeared at 1750-1400 and $2916 \mathrm{~cm}^{-1}$ were due to the carboxyl $\mathrm{C}=\mathrm{O}$ stretching vibration and the $\mathrm{C}-\mathrm{H}$ stretching vibration, respectively $[19,20]$. The FT-IR results indicated that the carboxyl group were successfully introduced into the surface of the CNTs after acidification. 


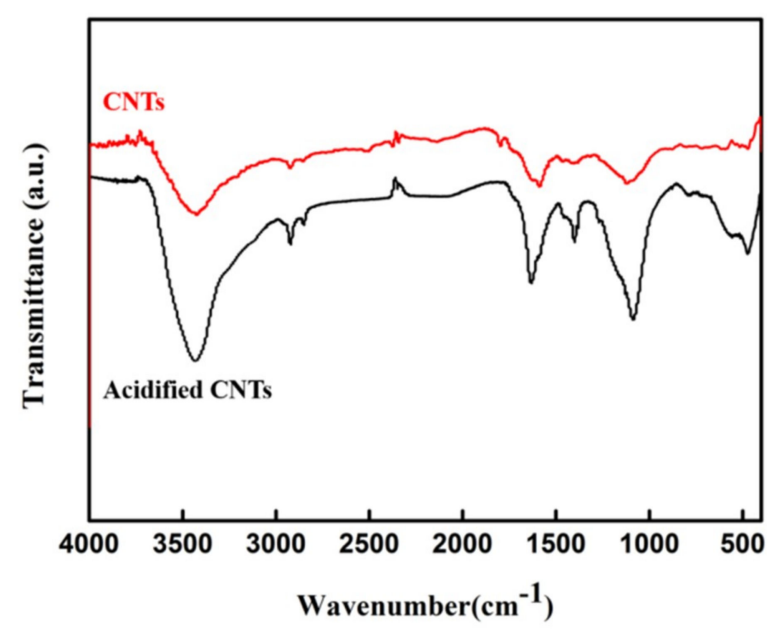

Figure 1. FT-IR patterns of CNTs before and after acidification.

\subsection{Characterization and Properties of the CNTs Modified BFs}

Surface Morphology of the CNTs Modified BFs

Figure 2 shows the surface morphology of the BFs modified with different mass fractions of CNTs ( $0.1 \mathrm{wt} . \%, 0.3 \mathrm{wt} . \%, 0.5 \mathrm{wt} . \%$, and $0.7 \mathrm{wt} . \%)$. We also inserted the zoomed-in figures on the right corner with higher magnifications to show the distribution in smaller scale. It can be seen in Figure 2 that, with the addition of the CNTs, the amount of the CNTs adhesion on BFs surface gradually increased. The CNTs can evenly distribute on the surface of BFs, as shown in Figure 2a-c. However, with the further addition of the CNTs, the effect of hydrogen bonding and electrostatic attraction on the surface was getting stronger [21], hence, the agglomeration happened on the surface of the BFs.
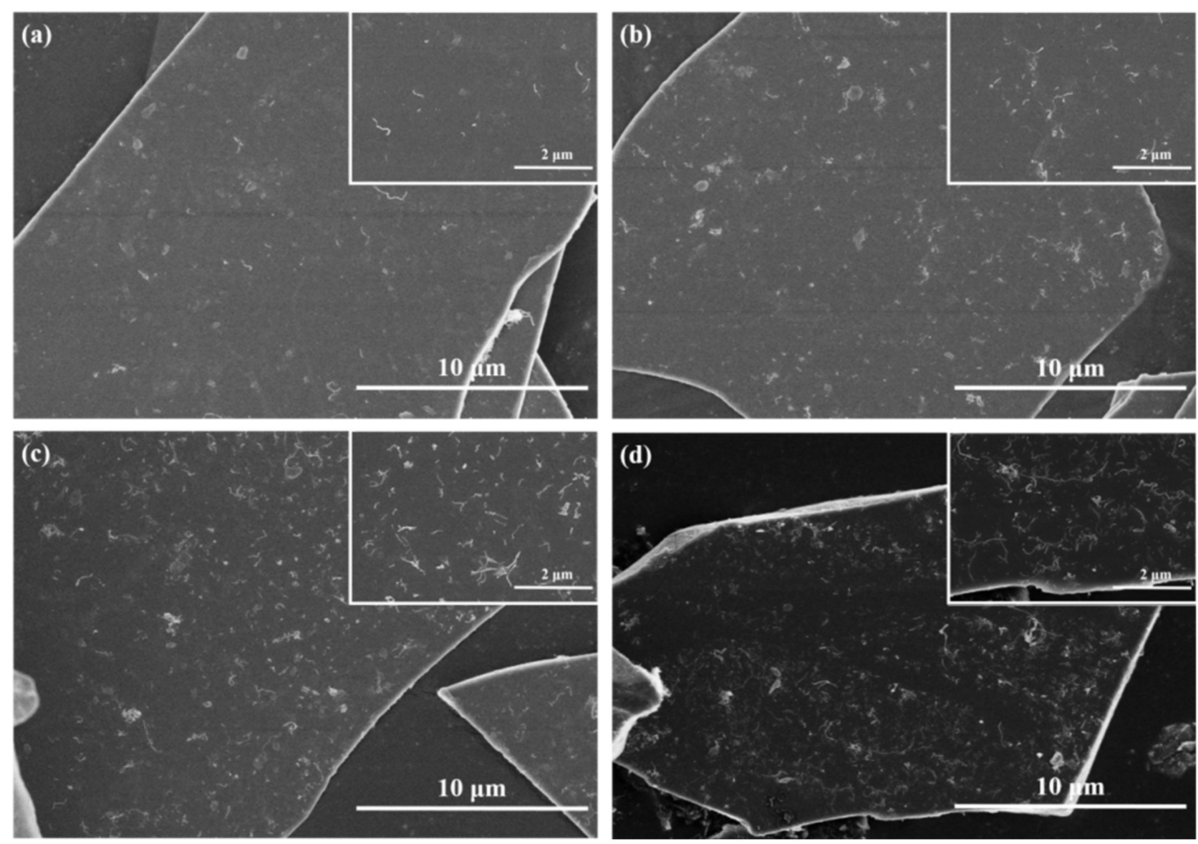

Figure 2. SEM images of basalt flakes modified with CNTs in different mass fractions. (a) 0.1 CNT-BF; (b) 0.3 CNT-BF; (c) 0.5 CNT-BF; (d) 0.7 CNT-BF. 


\subsection{Characterization and Properties of the CNT-BF/EP Coating}

\subsubsection{The Cross-Section Morphology of CNT-BF/EP Coating}

The morphology of the cross-section of the CNT-BF/EP coating, which is a fracture surface quenching by liquid nitrogen, is shown in Figure 3. The SEM images show that the bonding situation was gradually improved with the addition of the CNTs. Particularly, when the content of CNTs was 0.5 wt.\%, in Figure 3d, the modified BFs and resin had an obvious traction effect, which resulted in no obvious gap in the interface of the CNT-BF/EP coating fracture surface. However, according to Figure $3 \mathrm{e}$, the voids and micro-cracks can be observed in the fracture surface which can link to the agglomeration of CNTs.
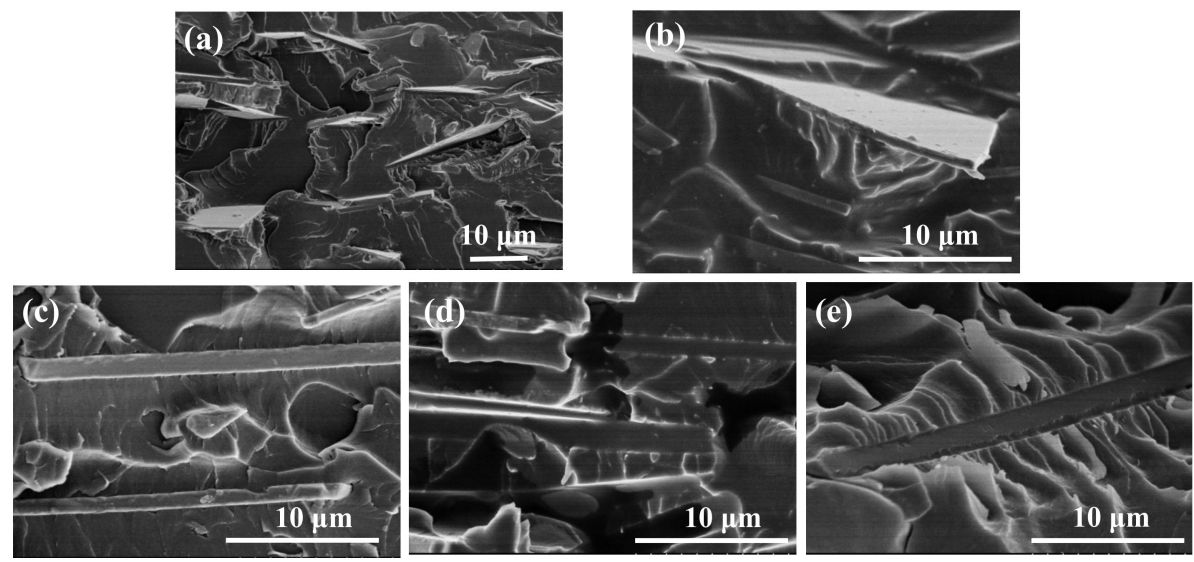

Figure 3. SEM images of the section morphology of CNT-BF/EP coating. (a) BF/EP; (b) $0.1 \mathrm{CNT}-\mathrm{BF} / \mathrm{EP}$;

(c) $0.3 \mathrm{CNT}-\mathrm{BF} / \mathrm{EP}$; (d) $0.5 \mathrm{CNT}-\mathrm{BF} / \mathrm{EP}$; (e) $0.7 \mathrm{CNT}-\mathrm{BF} / \mathrm{EP}$.

\subsubsection{The Tensile Strength Test of the CNT-BF/EP Coating}

It can be seen in Figure 4 that the tensile strength of the CNT-BF/EP coating increased significantly with the addition of CNTs. The tensile strength of the CNTs-BF/EP coating reaches a maximum of 30.3 MPa with the addition of $0.5 \mathrm{wt}$.\% CNTs. The three-dimensional structure formed by CNTs and BF, which can be seen in Figure 3, was well dispersed in the resin system which could diffuse the external force and reduce the phenomenon of stress concentration, thereby improving the mechanical properties of the CNT-BF/EP coating [22].

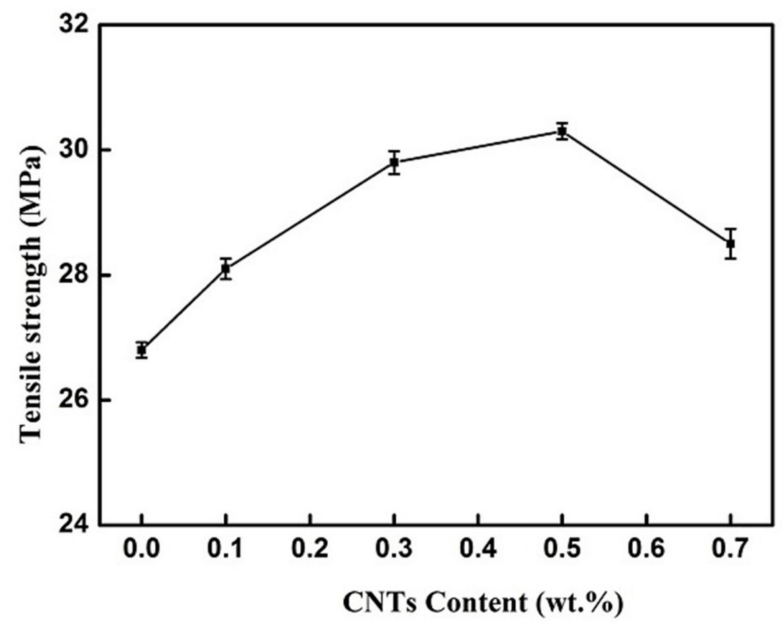

Figure 4. Tensile strength of CNT-BF/EP modified by different additions of the CNTs. 


\subsubsection{The Chemical Durability of the CNT-BF/EP Coating}

As shown in Figure 5, the weight loss rate of the coating after acid or alkali corrosion first decreased and then increased with the addition of CNTs. When the CNTs content was $0.5 \mathrm{wt} . \%$, the coating showed the best acid and alkali resistance (with the surface weight loss rate of $1.9 \mathrm{wt} . \%$ in the alkali solution and only $1.0 \mathrm{wt} . \%$ in the acid solution). A CNTs addition above $0.5 \mathrm{wt} . \%$ causes aggregation phenomena, which lead to a certain number of voids and micro-cracks (Figure 3e) on the surface of the CNT-BF/EP coating.

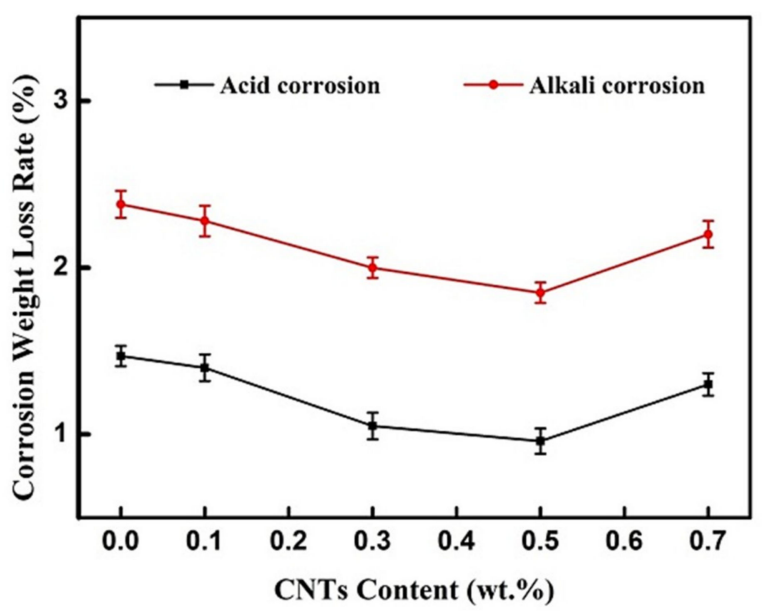

Figure 5. The weight loss rate of the CNT-BF/EP coating in $3 \mathrm{~mol} / \mathrm{L} \mathrm{HCl}$ and $3 \mathrm{~mol} / \mathrm{L} \mathrm{NaOH}$ solutions.

\subsubsection{Water Absorption of the CNT-BF/EP Coating}

Figure 6 shows the water absorption of the CNT-BF/EP coating was significantly reduced by the addition of the CNTs. The sample modified by $0.5 \mathrm{wt} . \%$ CNTs had the lowest water absorption after immersing in water for 24,240 , and $480 \mathrm{~h}$ at room temperature. The water-blocking ability of the sample modified by $0.7 \mathrm{wt} . \%$ CNTs was decreased to a similar level to that of the sample modified by 0.1 wt. $\%$ CNTs.

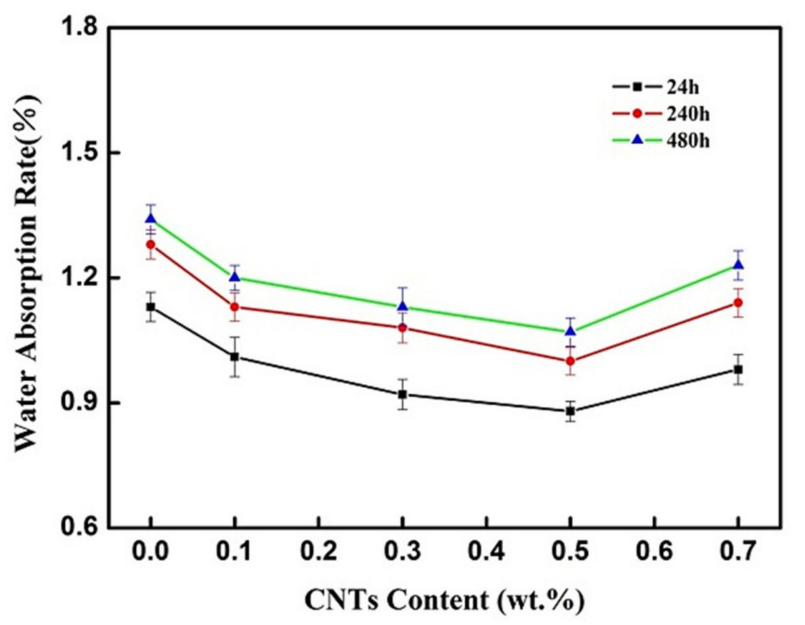

Figure 6. Water absorption of the CNT-BF/EP Coating by different additions of the CNTs. 


\section{Discussion}

The addition of the carbon nanotubes can significantly improve the tensile strength and chemical durability of the CNT-BF/EP coating. Based on Reference [8], we assume that the CNTs are closely linked to the epoxy resin and BFs, which prevents the shedding and displacement. The acidification treatment successfully enhanced the surface's chemical activity by grafting the functional groups such as - $\mathrm{OH}$. The acidified CNTs with many surface active centers and a large number of functional groups can fully combine with the BFs and resin matrix to form a strong cross-linking system, which has a good reinforcing effect on the coating and makes water and gas difficult to penetrate. The epoxy resin shows strong adhesion to CNTs due to the existence of these large number of functional groups which directly improved mechanical and chemical properties of the CNT-BF/EP coating.

A certain amount of CNTs, i.e., $0.5 \mathrm{wt}$.\%, can modify the surface of BFs while the agglomeration happened when we further increase the addition of the CNTs. The CNTs have large surface energy, which indicates that they are in a thermodynamically unstable state [8]. The agglomeration of the CNTs on the surface of the BFs generates voids and micro-cracks which weakens the binding between the BFs and epoxy resin. The corrosive medium can first enter the weak (micro-crack) zone and attack the coating [23]. On the other hand, the agglomeration of the CNTs on the surface of the BFs re-increased the water absorption which is due to the increase of extra paths for water from the voids and micro-cracks. Thus, excess addition of the CNTs plays a negative role in the tensile strength, chemical durability, and water blocking of the BFs coating.

Indeed, the composition of water in oceans or rivers are much more complicated than the deion water we used in the water absorption test (Figure 6). The mixed ions, e.g., $\mathrm{Na}^{+}, \mathrm{Ca}^{2+}, \mathrm{SO}_{4}{ }^{2-}$, and $\mathrm{Cl}^{-}$, affect the water absorption rate during a long-term ion exchange process. The surface corrosion are more significant than in the deion water, while still slower than in pure acid or alkali solutions. Therefore, a good chemical durability of the CNT-BF/EP coating in acid and alkali solutions provides a glimpse of the anti-corrosion behavior in oceans or rivers.

Moreover, we are also aware that the CNTs absorb light and therefore heat the coating, which in turn causes the rapid weathering of the CNT-BF/EP coating especially in the presence of water. Further researches are needed on the effect of light on the chemical stability and mechanical properties of the CNT-BF/EP coating.

\section{Conclusions}

In this study, a carbon nanotubes-modified basalt flake epoxy resin (CNT-BF/EP) coating was successfully prepared. The results showed that the acidified CNTs can significantly improve the interface compatibility of the BFs and resin, and thus, increase the tensile strength, chemical durability, and water resistance. The CNT-BF/EP coating sample modified by $0.5 \mathrm{wt} \%$ CNTs exhibited the best comprehensive performance with (1) excellent chemical durability (surface weight loss rate of $1.9 \mathrm{wt} . \%$ in the alkali solution and only $1.0 \mathrm{wt}$ \% in the acid solution at room temperature for $480 \mathrm{~h}$ ), (2) low water infiltration (water absorption of $1.1 \mathrm{wt} . \%$ for $480 \mathrm{~h}$ ), and (3) good mechanical properties (tensile strength of approximately 30.3 MPa). This study proves the feasibility of using carbon nanotubes to modify the basalt flake epoxy resin coating and enhance chemical durability and mechanical properties.

Author Contributions: Methodology, L.L. and Q.W. (Qingwei Wang); Formal analysis, L.L., Q.W. (Qihui Wang), Q.M., J.L. and L.D.; Data curation, L.L., Q.W. (Qihui Wang), Q.M. and J.L.; Writing-Original draft preparation, L.L. and L.D.; Writing-Review and editing, Q.W. (Qihui Wang), L.D. and W.J.; Supervision, Q.W. (Qingwei Wang), L.D. and W.J.; Funding acquisition, L.L. and Q.W. (Qingwei Wang).

Funding: This work was supported by the National Natural Science Foundation of China (NSFC, No. 51873032) and the Initial Research Funds for Young Teachers of Donghua University.

Conflicts of Interest: The authors declare no conflict of interest. 


\section{References}

1. Zhang, S.H.; Li, M.X.; Yoon, J.H.; Cho, T.Y.; He, Y.Z.; Lee, C.G. Microstructure and corrosion resistance of Ni-based alloy laser coatings with nanosize $\mathrm{CeO}_{2}$ addition. Sci. Technol. Adv. Mater. 2008, 9, 35002. [CrossRef] [PubMed]

2. Tian, W.; Liu, L.; Meng, F.; Liu, Y.; Li, Y.; Wang, F. The failure behaviour of an epoxy glass flake coating/steel system under marine alternating hydrostatic pressure. Corros. Sci. 2014, 86, 81-92. [CrossRef]

3. Wang, G.; Yang, J. Influences of glass flakes on fire protection and water resistance of waterborne intumescent fire resistive coating for steel structure. Prog. Org. Coat. 2011, 70, 150-156. [CrossRef]

4. Sathiyanarayanan, S.; Azim, S.S.; Venkatachari, G. Corrosion protection coating containing polyaniline glass flake composite for steel. Electrochim. Acta 2008, 53, 2087-2094. [CrossRef]

5. Lv, X.; Li, X.; Li, N.; Zhang, H.; Zheng, Y.-Z.; Wu, J.; Tao, X. ZrO 2 nanoparticle encapsulation of graphene microsheets for enhancing anticorrosion performance of epoxy coatings. Surf. Coat. Technol. 2019, 358, 443-451. [CrossRef]

6. Kim, S.H.; Heo, Y.-J.; Park, M.; Min, B.-G.; Rhee, K.Y.; Park, S.-J. Effect of hydrophilic graphite flake on thermal conductivity and fracture toughness of basalt fibers/epoxy composites. Compos. Part B Eng. 2018, 153, 9-16. [CrossRef]

7. Broughton, W.; Lodeiro, M.; Pilkington, G. Influence of coupling agents on material behaviour of glass flake reinforced polypropylene. Compos. Part A Appl. Sci. Manuf. 2010, 41, 506-514. [CrossRef]

8. Luo, L.; Ma, Q.; Wang, Q.; Ding, L.; Gong, Z.; Jiang, W. Study of a nano-SiO 2 microsphere-modified basalt flake epoxy resin coating. Coatings 2019, 9, 154. [CrossRef]

9. Yan, J.; Shi, J.; Zhang, P.; Tian, W.; Zhang, Y.; Sun, Z. Preparation and properties of epoxy/basalt flakes anticorrosive coatings. Mater. Corros. 2018, 69, 1669-1675. [CrossRef]

10. Song, P.-A.; Yang, H.-T.; Fu, S.-Y.; Wu, Q.; Ye, J.-W.; Lu, F.-Z.; Jin, Y.-M. Effect of carbon nanotubes on the mechanical properties of polypropylene/wood flour composites: Reinforcement mechanism. J. Macromol. Sci. Part B 2011, 50, 907-921. [CrossRef]

11. Fronza, B.M.; Lewis, S.; Shah, P.K.; Barros, M.D.; Giannini, M.; Stansbury, J.W. Modification of filler surface treatment of composite resins using alternative silanes and functional nanogels. Dent. Mater. 2019, 35, 928-936. [CrossRef] [PubMed]

12. Xue, H.; Wang, G.; Hu, M.; Chen, B. Modification of wollastonite by acid treatment and alkali-induced redeposition for use as papermaking filler. Powder Technol. 2015, 276, 193-199. [CrossRef]

13. Zhang, W.; Zou, X.; Wei, F.; Wang, H.; Zhang, G.; Huang, Y.; Zhang, Y. Grafting $\mathrm{SiO}_{2}$ nanoparticles on polyvinyl alcohol fibers to enhance the interfacial bonding strength with cement. Compos. Part B Eng. 2019, 162, 500-507. [CrossRef]

14. Szakács, J.; Mészáros, L. Synergistic effects of carbon nanotubes on the mechanical properties of basalt and carbon fiber-reinforced polyamide 6 hybrid composites. J. Thermoplast. Compos. Mater. 2018, 31, 553-571. [CrossRef]

15. Siddiqui, N.A.; Sham, M.-L.; Tang, B.Z.; Munir, A.; Kim, J.-K. Tensile strength of glass fibres with carbon nanotube-epoxy nanocomposite coating. Compos. Part A Appl. Sci. Manuf. 2009, 40, 1606-1614. [CrossRef]

16. Ma, Q.; Ding, L.; Wang, Q.; Yu, Y.; Luo, L.; Li, H. Preparation and characterization of continuous fly ash derived glass fibers with improved tensile strength. Mater. Lett. 2018, 231, 119-121. [CrossRef]

17. Yang, H.; Wu, S.; Duan, Y.; Fu, X.; Wu, J. Surface modification of CNTs and enhanced photocatalytic activity of $\mathrm{TiO}_{2}$ coated on hydrophilically modified CNTs. Appl. Surf. Sci. 2012, 258, 3012-3018. [CrossRef]

18. Gómez, S.; Rendtorff, N.M.; Aglietti, E.F.; Sakka, Y.; Suarez, G. Intensity of sulfonitric treatment on multiwall carbon nanotubes. Chem. Phys. Lett. 2017, 689, 135-141. [CrossRef]

19. Kim, S.D.; Kim, J.W.; Im, J.S.; Kim, Y.H.; Lee, Y.S. A comparative study on properties of multi-walled carbon nanotubes (MWCNTs) modified with acids and oxyfluorination. J. Fluor. Chem. 2007, 128, 60-64. [CrossRef]

20. Osorio, A.; Silveira, I.; Bueno, V.; Bergmann, C. $\mathrm{H}_{2} \mathrm{SO}_{4} / \mathrm{HNO}_{3} / \mathrm{HCl}$-Functionalization and its effect on dispersion of carbon nanotubes in aqueous media. Appl. Surf. Sci. 2008, 255, 2485-2489. [CrossRef]

21. Li, S.; Li, Z.; Ke, B.; He, Z.; Cui, Y.; Pan, Z.; Li, D.; Huang, S.; Lai, C.; Su, J. Magnetic multi-walled carbon nanotubes modified with polyaluminium chloride for removal of humic acid from aqueous solution. J. Mol. Liq. 2019, 279, 241-250. [CrossRef] 
22. Liang, C.; Song, P.; Ma, A.; Shi, X.; Gu, H.; Wang, L.; Qiu, H.; Kong, J.; Gu, J. Highly oriented three-dimensional structures of $\mathrm{Fe}_{3} \mathrm{O}_{4}$ decorated CNTs/reduced graphene oxide foam/epoxy nanocomposites against electromagnetic pollution. Compos. Sci. Technol. 2019, 181, 107683. [CrossRef]

23. Xu, Z.; Dai, J.; Niu, J.; He, L.; Mu, R.; Wang, Z. Isothermal oxidation and hot corrosion behaviors of diffusion aluminide coatings deposited by chemical vapor deposition. J. Alloy. Compd. 2015, 637, 343-349. [CrossRef] 